\title{
Der Einfluß von Gold auf den durch Parathormon gesteigerten Kollagenabbau der Rattenhaut ${ }^{1}$ )
}

\author{
Von H. Burkhardt, K. Rommel, F. Burkhardt und D. Welter \\ Aus der Seletion für Klinische Cbemie (Doz. Dr. K. Rommel) des Zentrums für Innere Medizin und Kinderbeilkunde der \\ Universität Ulm - Medizinisch Naturivissenschaftliche Hocbscbule -
}

(Eingegangen am 19. November 1970)

Es wurde der Einfluß von Gold auf den durch Parathormon gesteigerten Kollagenabbau in der Haut der Ratte untersucht. Die bei den Kontrolltieren durch Parathormon-Überdosierung hervorgerufene Verminderung des Gesamtkollagengehaltes der Haut konnte durch Vorbehandlung mit Gold verhindert werden. Es wurde aber eine starke Verminderung der in 0,45 $\mathrm{MaCl}$ löslichen Fraktion bei diesen Tieren gefunden.

\section{The effect of gold on the increased degradation of collagen caused by parathormone in rat skin}

The effect of gold on the increased degradation of collagen caused by parathormone in rat skin was studied. The decrease in the collagen content of the skin of control animals following the administration of parathormone was prevented by pretreatment with gold. There was a marked decrease, however, in the in $0.45 \mathrm{M} \mathrm{NaCl}$ soluble fraction in the gold-treated animals.

Die Bestimmung des Hydroxyprolingehaltes des Gewebes erlaubt die Berechnung des Kollagengehaltes. Die Bestimmung der löslichen Kollagenfraktionen gestattet unter Berücksichtigung des gesamten Kollagengehaltes Rückschlüsse auf Synthese- und Abbauvorgänge zu ziehen.

\section{Der Einfluß von Gold auf das Kollagen}

ADAM und Mitarbeiter (1, 2, 3) konnten mit Hilfe physikalisch-chemischer Methoden eine stärkere Vernetzung des Kollagens nach der Anwendung von Natrium-Gold-Thiosulfat in vivo nachweisen. Nach längerer Goldbehandlung fanden sie elektronenoptisch einen Einbau von Goldatomen in vivo und vitro. Mit einer Goldbehandlung konnte ein tierexperimentell erzeugter Lathyrismus in gewissem Umfang verhindert werden (2). Auch mit anderen Schwermetallen konnten ähnliche chemisch-physikalische Effekte erzielt werden (3). Die hier zitierten Untersuchungen wurden am Rattenschwanzknorpel durchgeführt. Am Rattenknochen und der Rattenhaut fand man unter dem Einfluß von Gold eine deutliche Verminderung des normalen Kollagenabbaus (4).

Vom Parathormon ist bekannt, daß es den KollagenAbbau fördert. Dementsprechend fand man bei zahlreichen Untersuchungen einen starken Anstieg der Hydroxyprolinausscheidung im Urin (5-10). Mit Isotopenuntersuchungen konnte man eindeutig den erhöhten Kollagenabbau sowohl von löslichem als auch unlöslichem Kollagen nachweisen $(11,12)$.

Daneben wurde gleichzeitig ein leicht vermehrter Kollagenaufbau gefunden (11-13).

1) Mit Unterstützung der Deutschen Forschungsgemeinschaft Bad Godesberg.
Die vorliegende Untersuchung beschäftigt sich mit der Frage, ob durch Gold ein durch Parathormonüberdosierung ausgelöster Anstieg des Kollagenabbaus verhindert werden kann.

\section{Untersuchungsmaterial und Methoden}

Untersucht wurden vier Gruppen von jeweils zehn Tieren:

1. Normaltiere

2. goldbehandelte Tiere

3. parathormonbehandelte Tiere

4. parathormon- und goldbehandelte Tiere.

Als Goldpräparat verwendeten wir ein Aurothiopolypeptid mit etwa $13 \%$ organisch gebundenem Gold (Auro-Detoxin, Fa. J. A. Wülfing, Düsseldorf).

Die Goldbehandlung der Gruppen 2 und 4 erstreckte sich über 15 Wochen, wobei zweimal wöchentlich $10 \mathrm{mg}$ Aurothiopolypeptid/kg Körpergewicht intramuskulär injiziert wurden.

Parathormon (Eli Lilly Comp. Indianapolis) erhielten die Gruppen 3 und 421 Tage lang in der Dosierung von 50 U.S.P.Einherten täglich intraperitoneal.

Nach Beendigung der Behandlung wurden die Tiere in leichter Äthernarkose durch Entbluten getötet. Das Serum wurde zur Bestimmung von Kreatinin verwendet. Die Haut über der rechten Skapula wurde in einer Ausdehnung von $2 \times 3 \mathrm{~cm}$ abrasiert und von der Subkutis abpräpariert. Sie wurde in kleine Stücke geschnitten und danach im Homogenisator Ultra-Turrax (Firma Kunkel) $9 \times 5$ Sek. mit Pausen von jeweils 55 Sek. in absol. Alkohol im Eisbad zerkleinert. Nach 2 Stdn. Stehen in absol. Alkohol bei $4^{\circ}$ wurde zentrifugiert und der Überstand abgesaugt. Das Sediment wurde zweimal mit Accton geschüttelt, zentrifugiert und der Uberstand jeweils abgesaugt. Nach Lufttrocknung des Gewebes wurde es abgewogen und zur Bestimmung von Hydroxyprolin im Gesamtkollagen sowie zur Gewinnung einer in $0,45 \mathrm{M} \mathrm{NaCl}$ löslichen Kollagenfraktion geteilt. Diese wurde durch ein 12 stdg. Schütteln in $5 \mathrm{~m} l$ der Salzlösung bei $4^{\circ}$ gewonnen. Die Hydrolyse erfolgte mit $1 \mathrm{ml} 32$ proz. $\mathrm{HCl}$ über $12 \mathrm{Stdn}$. bei $115^{\circ}$. Die Bestimmung des Hydroxyprolins erfolgte in Abänderung der Methode von STEgranN (14) mit dem Autoanalyzer (15). Das Kreatinin im Serum wurde mit der üblichen JAFFÉReaktion bestimmt. Die statistische Signifikanz der Veränderung wurde mit dem STUDENr-t-Test geprüft. 
Tab. 1

Hydroxyprolingehalt ( $\mu \mathrm{g} / \mathrm{mg}$ Gewebe der Haut unter Gold-, Parathormon- und kombinierter Gold-Parathormonbehandlung

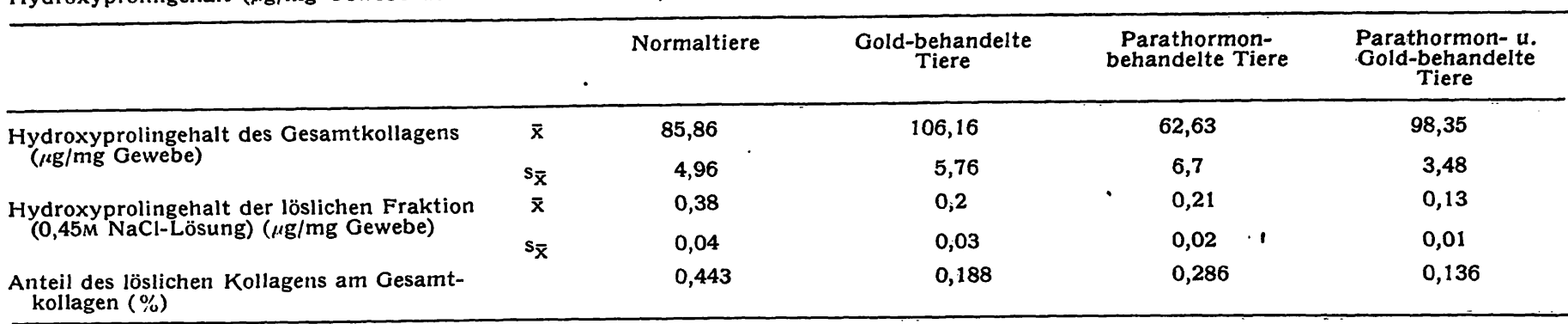

Tab. 2

Signifikanzen der Unterschiede des Hydroxyprolingehaltes der Haut unter Gold-, Parathormon- und kombinierter Gold-Parathormonbehandlung

\begin{tabular}{|c|c|c|c|c|c|c|}
\hline Verglichen mit & $\begin{array}{c}\text { Normaltiere } \\
\text { Goldbehandelten } \\
\text { Tieren } \\
\mathrm{p}<\end{array}$ & $\begin{array}{c}\text { Normaltiere } \\
\text { Parathormonbe- } \\
\text { handelten } \\
\text { Tieren } \\
\text { p < }\end{array}$ & $\begin{array}{c}\text { Normaltiere } \\
\text { Parathormon- u. } \\
\text { Goldbehandelten } \\
\text { Tieren } \\
\text { p < }\end{array}$ & $\begin{array}{c}\text { Goldbehandelte } \\
\text { Tiere } \\
\text { Parathormonbe- } \\
\text { handelten } \\
\text { Tieren } \\
\text { p < } \\
\end{array}$ & $\begin{array}{c}\text { Goldbehandelte } \\
\text { Tiere } \\
\text { Parathormon- } u . \\
\text { Goldbehandelten } \\
\text { Tieren } \\
\text { p < }\end{array}$ & $\begin{array}{c}\text { Parathormon } \\
\text { behandelte Tiere } \\
\text { Parathormon- } u . \\
\text { Goldbehandelten } \\
\text { Tieren } \\
\text { p }<\end{array}$ \\
\hline $\begin{array}{l}\text { Hydroxyprolingehalt des Ge- } \\
\text { samtkollagens }\end{array}$ & 0,01 & 0,01 & 0,05 & 0,0005 & $0,1^{\circ}$ & 0,0005 \\
\hline $\begin{array}{l}\text { Hydroxyprolingehalt der } \\
\text { Jöslichen Fraktion }(0,45 \mathrm{M} \\
\mathrm{NaCl} \text {-Lösung) }\end{array}$ & 0,0025 & 0,0025 & 0,0005 & 0,4 & 0,01 & 0,01 \\
\hline
\end{tabular}

\section{Ergebnisse und Diskussion}

Die Ergebnisse sind aus den Tabellen 1 und 2 ersichtlich.

Für eine Nierenschädigung der behandelten Tiere bestand kein Anhalt, die Kreatininkonzentration im Serum betrug in der Normalgruppe im Mittel $0,67 \mathrm{mg} /$ $100 \mathrm{ml}( \pm 0,05)$, nach alleiniger Goldbehandlung $0,64 \mathrm{mg} / 100 \mathrm{ml}( \pm 0,06)$, nach Parathormon $0,63 \mathrm{mg} /$ $100 \mathrm{ml}( \pm 0,03)$ und nach Parathormon- und Goldbehandlung $0,66 \mathrm{mg} / 100 \mathrm{ml}( \pm 0,04)$. (In Klammern ist die einfache Standardabweichung angegeben.)

Nach Goldbehandlung trat ein signifikanter Anstieg des Gesamtkollagens der Haut auf, während die in $0,45 \mathrm{M} \mathrm{NaCl}$ lösliche Kollagenfraktion abnahm. Parathormon steigerte den Abbau des löslichen und unlöslichen Kollagens, wie es besonders auch bei einer
Untersuchung von LAIrineN (11) gefunden wurde. Dieser Parathormoneffekt auf das unlösliche Kollagen läßt sich bei den goldbehandelten Tieren nicht nachweisen. Auffällig war aber die starke Verminderung der löslichen Kollagenfraktion, die im Vergleich zu den nur mit Gold und nur mit Parathormon behandelten Tieren signifikant erniedrigt ist. Wahrscheinlich addieren sich hier zwei Effekte. Im Gegensatz zum unlöslichen Kollagen der goldbehandelten Tiere ist die lösliche Kollagenfraktion durch Parathormon angreifbar, so daß ihr verstärkter Abbau erfolgt. Andererseits ist die Bildung von löslichem Kollagen durch verminderten Abbau des unlöslichen Kollagens herabgesetzt. Insgesamt etlauben diese Untersuchungsergebnisse den Schluß, daß ein durch Parathormon gesteigerter Kollagenabbau durch eine Goldbehandlung vermindert werden kann.

\section{Literatur}

1. Adam, M., Ann. Rheumat. Dis., London 24, 378 (1965). 2. AdAM, M. und K. KüH, European J. Biochem. 3, 407 (1968). 3. Adam, M., P. Fietzek, Z. Deil, S. Rosmus und K. KühN, European J. Biochem. 3, 415 (1968). - 4. BUR KHARDT, H., K. ROMMEL, F. BuRKhaRdT und D. WeLter, Z. Rheumaforsch., im Druck. 5. Dull, T. A. und P. H. Henneman, N. England J. Med. 268, 132 (1963). - 6. Horwith, M., J. R. Buchanan, S. T. GMundsson und C. A. Mautalen, J. Clin. Endocr., Springfield 26, 885 (1966). - 7. Klein, L., K. Albertsen und P. H. Curtiss jr., Metabolism, Baltimore 11, 1023 (1962). - 8. Prockop, D. J. und K. I. Kivirikko, Ann. Int. Med. 66, 1243 (1967). - 9. LeE, C. A. und H. M. Lloxd, Med. J. Australia 1, 992 (1965). 10. Johnstón, C. C. jr. und W. P. Derss jr., Metabolism, Baltimore 14, 523 (1965). - 11. Latrinen, O., Endocrinology 80, 815 (1967). - 12. Flanagan, B. und G. Nichols jr., J. Clin. Invest. 44, 1795 (1965). - 13. Flanagan, B. und G. Nichols jr., Endocrinology 74, 180 (1964). - 14. StegemanN, H., HoppeSeyler's Z. physiol. Chem. 311, 41 (1958). - 15. GRANT, R. A., Chemie Technikon Frankfurt 326 (1964).

Doz. Dr. K. Rommel

Univ. Ulm, Med.-Naturwissenschaftl. Hochschule Zentrum f. Inn. Med.

79 Ulm (Donau)

Steinhövelstr. 9 